\title{
Interactive virtual feedback improves gait motor imagery after spinal cord injury: An exploratory study
}

\author{
Meyke Roosink $^{\mathrm{a}}$, Nicolas Robitaille ${ }^{\mathrm{a}}$, Philip L. Jackson ${ }^{\mathrm{a}, \mathrm{b}}$, Laurent J. Bouyer ${ }^{\mathrm{a}, \mathrm{c}}$ \\ and Catherine Mercier ${ }^{\mathrm{a}, \mathrm{c}, *}$ \\ ${ }^{a}$ Center for Interdisciplinary Research in Rehabilitation and Social Integration (CIRRIS), Québec, QC, Canada \\ ${ }^{\mathrm{b}}$ School of Psychology, Laval University, Québec, QC, Canada \\ ${ }^{\mathrm{c}}$ Department of Rehabilitation, Faculty of Medicine, Laval University, Québec, QC, Canada
}

\begin{abstract}
.
Purpose: Motor imagery can improve motor function and reduce pain. This is relevant to individuals with spinal cord injury (SCI) in whom motor dysfunction and neuropathic pain are prevalent. However, therapy efficacy could be dependent on motor imagery ability, and a clear understanding of how motor imagery might be facilitated is currently lacking. Thus, the aim of the present study was to assess the immediate effects of interactive virtual feedback on motor imagery performance after SCI.

Methods: Nine individuals with a traumatic SCI participated in the experiment. Motor imagery tasks consisted of forward (i.e. simpler) and backward (i.e. more complex) walking while receiving interactive versus static virtual feedback. Motor imagery performance (vividness, effort and speed), neuropathic pain intensity and feasibility (immersion, distraction, side-effects) were assessed.

Results: During interactive feedback trials, motor imagery vividness and speed were significantly higher and effort was significantly lower as compared static feedback trials. No change in neuropathic pain was observed. Adverse effects were minor, and immersion was reported to be good.

Conclusions: This exploratory study showed that interactive virtual walking was feasible and facilitated motor imagery performance. The response to motor imagery interventions after SCI might be improved by using interactive virtual feedback.
\end{abstract}

Keywords: Spinal cord injury, visual feedback, virtual reality therapy, gait, chronic pain

\section{Introduction}

Motor imagery is the mental execution of a movement without the production of actual movement. Interestingly, motor imagery presents many similarities with movement execution and both are thought to share, at least in part, similar underlying mechanisms

${ }^{*}$ Corresponding author: Catherine Mercier, Center for interdisciplinary research in rehabilitation and social integration (CIRRIS), 525 Boul. Hamel, Québec, QC, G1M 2S8, Canada. Tel.: +1 418529 9141/Ext. 6701; Fax: +1 418529 3548; E-mail: catherine.mercier@rea.ulaval.ca.
(Decety, 1996; Jackson, Lafleur, Malouin, Richards, \& Doyon, 2001; Jeannerod, 2001). As a rehabilitation intervention, motor imagery could improve motor function after cerebral or spinal cord lesions, and has been used in the treatment of neuropathic pain (Bowering et al., 2013; Malouin \& Richards, 2010; Malouin, Saimpont, Jackson, \& Richards, 2013; Mulder, 2007). Whereas its therapeutic effect on motor function has been ascribed to the strengthening of motor programs by repeated activation of motor representations (Mulder, 2007), the mechanisms underlying pain reduction are less clear. It has 
been proposed that motor imagery could normalize the complex interaction between mental body representations, sensorimotor integration, nociception, and pain (Moseley \& Flor, 2012; Moseley, Gallace, $\&$ Spence, 2012).

The simultaneous effects of motor imagery on motor function and pain make it a particularly relevant intervention for individuals with spinal cord injury (SCI) (Roosink \& Mercier, 2014; Villiger, Bohli, et al., 2013), in whom neuropathic pain is prevalent (ranging between $34 \%$ and $83 \%$ ) and is considered particularly difficult to treat (Siddall, McClelland, Rutkowski, \& Cousins, 2003; Turner, Cardenas, Warms, \& McClellan, 2001). Importantly, pain interferes with the rehabilitation process and with motor recovery. For example, the presence of pain has been associated with lower levels of physical activity (Tawashy, Eng, Lin, Tang, \& Hung, 2009) and of community reintegration (Donnelly \& Eng, 2005), as well as with impaired motor learning (Baumbauer, Young, \& Joynes, 2009; Boudreau et al., 2007; Bouffard, Bouyer, Roy, \& Mercier, 2014; Lamothe et al., 2014).

In contrast to other neurological patient populations, the number of studies that investigated motor imagery in individuals with SCI is still relatively small (Di Rienzo, Collet, Hoyek, \& Guillot, 2014; Malouin \& Richards, 2010). Moreover, given that motor imagery has often been applied in combination with other therapeutic modalities, such as visual feedback (e.g. mirror therapy, virtual reality) (Villiger, Bohli, et al., 2013) or physical exercise (Sharp et al., 2014), the contribution of each modality to therapy efficacy remains largely unclear (Roosink $\&$ Mercier, 2014). A particular therapeutic approach that has been studied in individuals with neuropathic pain after SCI has initially been called "virtual walking" (Moseley, 2007). In these studies, participants performed gait imagery while observing their own upper body in a mirror, but their lower limbs were "replaced" by a video projection of walking legs (Kumru et al., 2013; Moseley, 2007; Soler et al., 2010). Patients were encouraged to imagine themselves walking and, when possible, to move their arms and upper body in synchrony with the rhythm of the projection to increase the illusion's effectiveness. Only effects on neuropathic pain were studied, and mixed results were obtained (Kumru et al., 2013; Moseley, 2007; Soler et al., 2010). For example, different effects were observed at different time-points during treatment for paroxysmal pain, mechanical allodynia and dysesthesias (Soler et al., 2010). Motor imagery has also been combined with transcranial direct current stimulation (tDCS), and this combination seemed to be associated with additional reductions in pain combined with either intervention alone (Kumru et al., 2013; Soler et al., 2010). To make matters even more complex, individuals with SCI sometimes reported increased pain while performing motor imagery (Gustin et al., 2008). This might be explained by the fact that some participants have difficulties imagining movements (Mercier, 2012; Raffin, Giraux, \& Reilly, 2013). As such, a certain ability to perform motor imagery might be needed in order to obtain beneficial effects (Bowering et al., 2013; Moseley, 2006). Although the triggering of initial pain (i.e. within session) has not been shown to be detrimental to the long term effects of motor imagery or virtual feedback therapy (Beaumont, Mercier, Michon, Malouin, \& Jackson, 2011; Mercier \& Sirigu, 2009), therapeutic efficacy might be improved by facilitating motor imagery (Roosink \& Mercier, 2014).

We recently developed a virtual walking system that can be interactively controlled, providing realtime motion-dependent visual feedback. Briefly, the system uses movement tracking (arm swing) to control a full-body avatar that can walk forward or backward in a realistic virtual scene. As such, and in contrast to previous studies, this system allows participants to control the initiation and the speed at which their virtual image is walking, i.e. the avatar is synchronized with the subject's motor imagery speed rather than the reverse. In addition, arm kinematics can be used as an objective measurement of task performance, e.g. imagery speed.

The primary aim of this proof-of-principle study was to use this virtual walking system to assess the immediate effects of interactive virtual feedback on motor imagery performance (vividness, effort, speed) during forward (i.e. simpler) and backward (i.e. more complex) gait imagery in individuals with SCI. The secondary aims were to explore changes in neuropathic pain intensity in the participants with neuropathic pain, and to assess the feasibility of the approach (immersion, distraction, side-effects). Another secondary aim was to compare the effect of anodal and cathodal tDCS applied bilaterally over the bilateral lower limb area of the primary motor cortex. However, the final sample size did not allow testing the effects of tDCS; this aspect will therefore not be described in details in the present paper. We hypothesized that motor imagery would be more vivid and faster, and would require less mental effort when combined with interactive virtual feedback. 


\section{Methods}

\subsection{Participants}

A convenience sample was recruited among the outpatients of the Institut de réadaptation en déficience physique de Québec (IRDPQ). Individuals were eligible for participation if they were at least 18 years old, had sustained a traumatic SCI at least 3 months prior to participation, and had a lesion at the level of $\mathrm{C} 4$ or lower. Recruitment was performed by contacting participants from previous SCI research projects and by physiatrist referral. Potential participants were excluded if they had insufficient trunk balance or arm motor function to perform the task, psychiatric (e.g. psychosis), psychological (e.g. cognitive) or neurological disorders other than SCI (e.g. stroke) that might interfere with motor imagery performance, non-corrected vision impairments, contraindications for tDCS or pregnancy. The project was approved by the IRDPQ ethics review board (\#2013-322). All participants received written and oral information, and provided written informed consent prior to participation.

\subsection{Interactive virtual walking system}

The interactive set-up consisted of an inertial movement sensor, a virtual reality system, two projectors (allowing for 3D vision), and a large silver-coated projection screen (see Fig. 1 for details). The virtual scene displayed a forest path leading to the door of a small cabin (fixed distance) and a horizontal progress bar in the lower left corner of the screen (i.e. to provide feedback on the distance covered). Interactive virtual walking was performed under 4 different conditions: 1) forward with avatar (FWA), 2) forward with a static virtual scene (FWS), 3) backward with avatar (BWA), and 4) backward with a static virtual scene (BWS). During all conditions, the progress bar was updated in real-time based on right upper arm swing in the sagittal plane measured with the inertial movement sensor. During avatar trials, the same sensor data was used to additionally animate a realistic male avatar (as seen from the back) for forward (from the starting line to the cabin) or backward walking (from the cabin back to the starting line) and the virtual scene changed in real-time depending on the instructed walking direction (forward or backward).

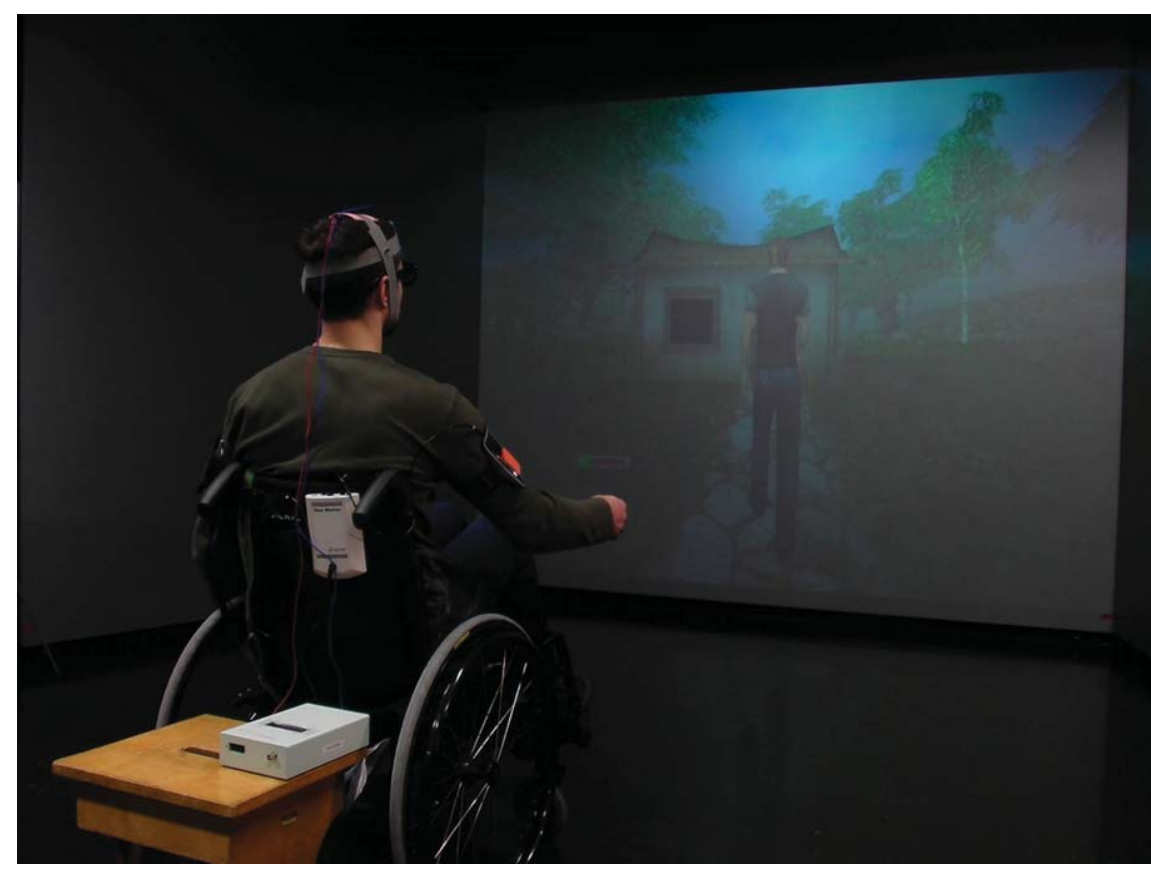

Fig. 1. The experimental set-up. An inertial sensor (MTx miniature inertial measurement unit, Xsens Technologies BV, Enschede, the Netherlands) was attached to the subject's right upper arm. A dummy sensor was attached to the left upper arm to avoid focusing on one arm. The virtual reality system was controlled by D-flow software (CAREN, Motek Medical BV, Amsterdam, the Netherlands), and the virtual scene was projected in 3D (2 projectors, CP-WX8255A, $1920 \times 1080$ High Definition, Hitachi, Tokyo, Japan) on a large silver-coated projection screen (projection surface $3.05 \mathrm{~m} \times 2.06 \mathrm{~m}$ ). The inertial sensor was connected to the virtual reality system via a wireless Bluetooth connection. The rotation data for the sagittal plane, acquired with the inertial sensor, was used to animate the progress bar in the lower left corner. In addition, during avatar trials the same signal was used to go through a pre-programmed walk-cycle animation of the avatar. 
During static trials, the distance progress bar was animated but the virtual scene remained static. Sagittal movement data of the right upper arm was stored for off-line analysis of motor imagery speed (task duration in seconds).

\subsection{Baseline session}

Prior to the experiment, demographic (gender, age) and medical data (ASIA impairment scale, lesion level, SCI onset, ambulation) were recorded. Pain characteristics were assessed using the Basic Pain Data Set (Widerstrom-Noga et al., 2014). Symptoms of anxiety and depression were assessed using the Hospital Anxiety and Depression Scale (HADS) (Zigmond \& Snaith, 1983). General motor imagery ability (dominant side only) was assessed using the Kinesthetic and Visual Imagery Questionnaire (KVIQ-10) (Malouin et al., 2007). Actual motor performance was replaced by a video animation displaying the movement to be imagined, to make the task similar across participants with different levels of impairment.

\subsection{Interactive virtual walking protocol}

The experiment consisted of two sessions (1.5 hours each, at least 1 week apart to avoid carry-over effects) of interactive virtual walking. Two sessions were originally planned to compare the addition of anodal or cathodal tDCS over M1 (lower limb representation; bilateral stimulation) but given that no difference or trend was observed between the two types of stimulation, the effects of tDCS were not studied further and data was pooled across sessions.

Participants were seated approximately 1.5 meters away from the screen, allowing for a real-size view of the avatar. They were equipped with 2 adjustable armbands each containing an inertial movement sensor (1 active [right] and 1 dummy sensor [left], to avoid focusing on one arm) and were wearing 3D glasses. They were instructed to perform gait imagery at a comfortable speed while swinging their arms according to their imagined gait speed, and while focusing on the bodily sensations produced by gait imagery. They practiced gait imagery in the virtual scene under all 4 conditions (only 2 trials per condition to avoid fatigue), and practiced rating the perceived motor imagery vividness $(0=$ no sensations, $100=$ sensations as intense as if actually performing the movement) and motor imagery effort
$(0=$ no effort, $100=$ extreme effort $)$, the current neuropathic pain intensity and the presence and intensity of other types of pain (rated independently; $0=$ no pain, $100=$ worst pain imaginable).

The actual experiment consisted of 12 trials ( 3 for each condition). The order of conditions was pseudo-randomized and counter-balanced across participants. After each trial, participants rated the perceived motor imagery vividness and effort and the intensity of pain as practiced (pseudo-randomized). There was a short break after each trial. Prior to and at the end of the experiment, the current neuropathic pain intensity was assessed. In addition, at the end of the experiment, the interaction with the avatar and virtual scene (immersion, distraction) was assessed on a 1-7 scale using a subset of questions from the Presence Questionnaire (see Supplementary Table 1) (Witmer \& Singer, 1998).

\subsection{Outcome measures}

Primary outcome measures were motor imagery vividness, effort and speed. Secondary outcome measures included ongoing neuropathic pain intensity (pre-post change), perceived interaction with the avatar and virtual environment (average immersion and distraction scores), and presence of side-effects.

\subsection{Statistical analyses}

Statistical testing was performed under SPSS 13.0 for Windows (SPSS Inc. Chicago, IL, USA) using an alpha threshold of 0.05 . For motor imagery performance, $2 \times 2$ repeated measures analyses of variance (ANOVA) were performed for each motor imagery outcome parameter with within-subject factors Visual Feedback (avatar, static) and Direction (forward, backward).

\section{Results}

\subsection{Demographics, medical data, and motor imagery ability}

The characteristics of the 9 participants are presented in Table 1. Seven participants reported neuropathic pain. Other types of pain were also reported (musculoskeletal $n=5$, visceral $n=1$, headache $n=1$ ), and several participants received pharmacological pain treatment (analgesics $n=5$, antidepressants $n=3$, anticonvulsants $n=6$ ). Based 
Table 1

Demographic and medical data

\begin{tabular}{|c|c|c|c|c|c|c|c|c|c|}
\hline ID & $\mathrm{M} / \mathrm{F}$ & Age (years) & AIS & SCI level & SCI onset (months) & Ambulation & NP (type, intensity) & HADS (A/D) & KVIQ \\
\hline$\overline{1}$ & $\mathrm{M}$ & 72 & $\mathrm{~A}$ & $\mathrm{~T} 4$ & 108 & Wheelchair & AL, NRS50 & $7 / 4$ & 10 \\
\hline 2 & M & 25 & A & C5-C6 & 103 & Wheelchair & None & $2 / 4$ & 35 \\
\hline 3 & M & 47 & A & C6-C7 & 14 & Wheelchair & BL, NRS20 & $3 / 5$ & 14 \\
\hline 4 & M & 52 & A & T5-T6 & 78 & Wheelchair & None & $0 / 4$ & 23 \\
\hline 5 & M & 51 & $\mathrm{~A}$ & T12-L1 & 29 & Wheelchair & AL+BL, NRS 80 & $9 / 7$ & 33 \\
\hline 6 & $\mathrm{~F}$ & 67 & $\mathrm{C}$ & L2-L3 & 56 & Walk ( 2 canes) & BL, NRS50 & $5 / 8$ & 45 \\
\hline 7 & M & 56 & $\mathrm{D}$ & C3-4-5 & 135 & Walk (no aid) & AL+BL, NRS60 & $9 / 6$ & 40 \\
\hline 8 & $\mathrm{~F}$ & 48 & $\mathrm{~A}$ & $\mathrm{~T} 8$ & 119 & Wheelchair & AL+BL, NRS50 & $7 / 6$ & 39 \\
\hline 9 & M & 56 & $\mathrm{D}$ & $\mathrm{T} 2$ & 85 & Walk ( 1 cane) & AL+BL, NRS50 & $6 / 7$ & 27 \\
\hline \multirow[t]{4}{*}{ ALL } & $2 \mathrm{~W}$ & $53 \pm 13$ & $6 \mathrm{~A}$ & $3 \mathrm{C}$ & $81 \pm 41$ & 3 Walk & 2 None & A $5 \pm 3$ & $30 \pm 12$ \\
\hline & $7 \mathrm{M}$ & & $1 \mathrm{C}$ & $5 \mathrm{~T}$ & & 6 Wheelchair & $1 \mathrm{AL}$ & D $6 \pm 2$ & \\
\hline & & & $2 \mathrm{D}$ & $1 \mathrm{~L}$ & & & $2 \mathrm{BL}$ & & \\
\hline & & & & & & & $4 \mathrm{AL}+\mathrm{BL}$ & & \\
\hline
\end{tabular}

ALL data is presented as number of subjects or as mean \pm SD. AIS: ASIA impairment scale (range A-D), A/D: anxiety/depression, AL: at-level neuropathic pain, BL: below-level neuropathic pain, F: female, HADS: hospital anxiety and depression scale (subscale range 0-21), KVIQ: kinesthetic and visual imagery questionnaire (range 10-50), M: man, NP: neuropathic pain.

on KVIQ scores, general motor imagery ability was found to be very variable across participants, ranging from very poor to very good.

\subsection{Motor imagery performance}

Motor imagery vividness, effort and speed are presented in Fig. 2. ANOVAs revealed significant effects of Visual Feedback $\left(\mathrm{F}_{1,8}=22.781, p=0.001\right)$ and Direction $\left(\mathrm{F}_{1,8}=7.519, p=0.025\right)$ for motor imagery speed, and of Visual Feedback for motor imagery vividness $\left(\mathrm{F}_{1,8}=5.859, p=0.042\right)$ and effort $\left(\mathrm{F}_{1,8}=6.905, p=0.030\right)$. No significant interaction effects were found. This indicates that the presence of the avatar was associated with improved motor imagery vividness and speed and with lower motor imagery effort compared to trials in which the static scene was displayed, and that participants imagined walking faster during forward as compared to backward trials.

\subsection{Neuropathic pain intensity}

The average change in neuropathic pain intensity from pre to post experiment was -2 (on a scale of $0-100$ ), and the $95 \%$ confidence interval included 0 (-6 to 2), indicating that neuropathic pain intensity did not significantly change during the experiment. One participant (ID5) reported that pain was reduced by $70 \%$ during the trials (i.e. while performing motor imagery), but increased back to pre-trial levels once the trials were finished.

\subsection{Feasibility and adverse effects}

The interaction with the avatar and virtual scene was generally reported to be good, the average scores for immersion questions across 2 sessions being $5.3 \pm 0.8$ (mean $\pm \mathrm{SD}$; 0-7 scale). Several participants with a complete lesion (ID1, ID4, ID8) reported being surprised to feel actual sensations in their legs during the task. In contrast, one participant (ID3) reported no sensations at all during interactive virtual walking. Distracters were generally considered to be minor, the average scores for distraction questions across 2 sessions being $2.4 \pm 0.8$, including perceived delays (mostly at the first step), avatar movements not corresponding with the participant's gait representation, and unnatural arm movements (e.g. to avoid wheelchair contact).

No major adverse events occurred. Negative sideeffects included increased transient musculoskeletal pain $(n=1)$, physical fatigue/effort $(n=4)$, and difficulties to maintain attention $(n=2)$.

\section{Discussion}

This exploratory study assessed the effect of interactive virtual feedback on motor imagery performance during virtual walking in individuals with SCI. We found that during trials in which an avatar could be interactively controlled, motor imagery vividness and speed were significantly higher and effort was significantly lower compared to trials in which only a static scene was displayed. No change in neuropathic pain 
A : Vividness

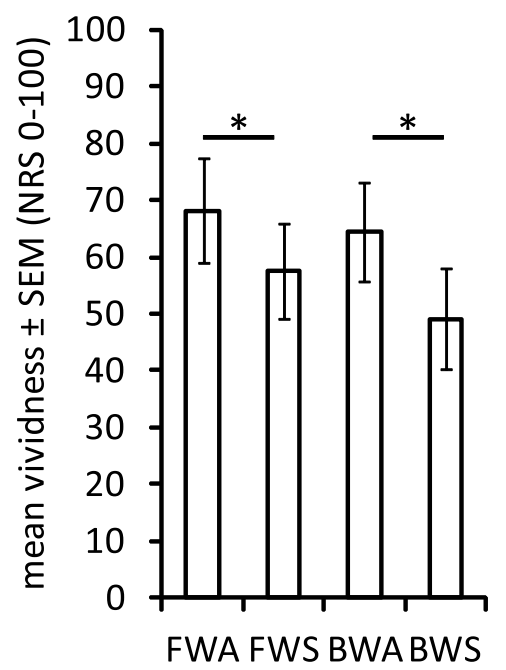

B : Effort

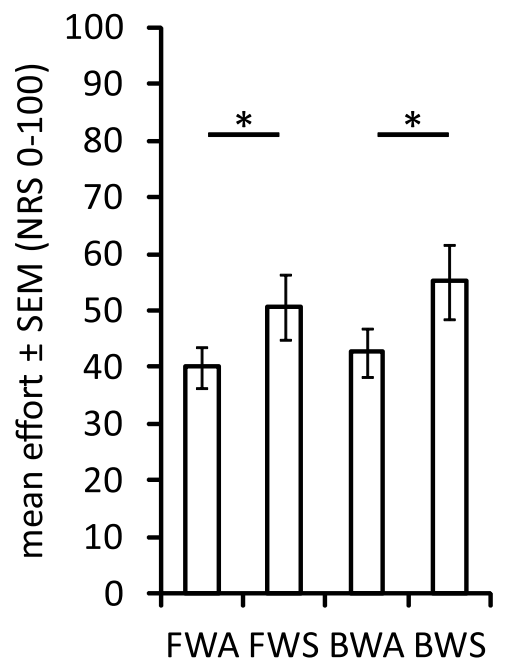

C: Speed

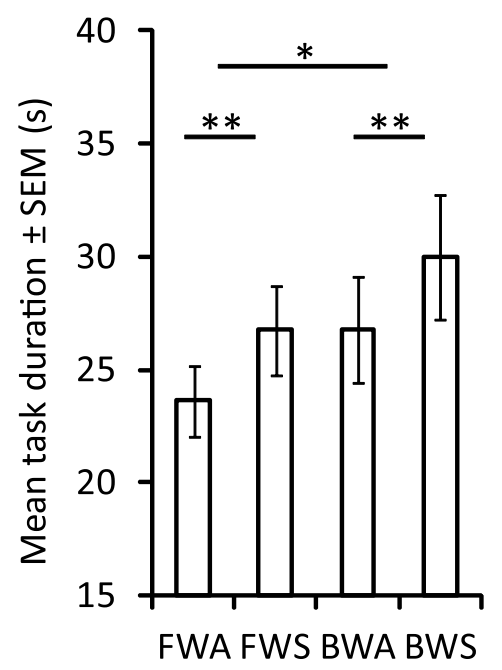

Fig. 2. Mean motor imagery vividness (A), effort (B) and speed (C) during interactive virtual walking. BWA: backward walking with avatar, BWS: backward walking with static image, FWA: forward walking with avatar, FWS: forward walking with static image, ${ }^{*} p<0.05$, ${ }^{* *} p<0.01$.

intensity was observed. Adverse effects were minor, and immersion was reported to be good.

\subsection{Interactive virtual feedback improved motor imagery performance}

Previous studies assessing the therapeutic effects of virtual walking (Kumru et al., 2013; Moseley, 2007; Soler et al., 2010) or other forms of motor imagery (Sharp et al., 2014; Sumitani et al., 2008; Villiger, Bohli, et al., 2013) on motor function and/or neuropathic pain in individuals with SCI have shown promising results (Roosink \& Mercier, 2014). Still, many aspects related to the use of motor imagery after SCI remain unclear, including the role of visual feedback (Roosink \& Mercier, 2014). The present study is thus an important extension to previous work as it explored two important elements relating to the impact of visual feedback on motor imagery performance: 1 ) the feasibility of using interactive (self-paced) visual feedback of gait; and 2) the added value of interactive versus static visual feedback.

Interactive virtual feedback was found to be feasible (i.e. overall immersion was high, despite a limited match between the avatar's and participant's physical appearance; overall distraction was low; adverse effects were minor), and demonstrated feedback-specific (avatar versus static) as well as task-specific (forward versus backward) differences in gait imagery performance. Although motor imagery abilities were variable across participants, all measures concurred to show an added value of interactive versus static feedback, regardless of gait direction (forward, backward). Whether this increase was due to the avatar, to the dynamic virtual scene, to a mismatch between visual and somatosensory feedback in the static scene, or to a combination of these factors remains to be addressed in future studies.

Several mechanisms could be responsible for the observed effects including sensorimotor and cognitive-emotional mechanisms. For example, interactive virtual feedback may provide additional information about interactions of the body with the environment (Betker, Desai, Nett, Kapadia, \& Szturm, 2007; Sayenko et al., 2010) and may lead to additional activation of brain areas involved in motor preparation and motor control (Mulder, 2007; Villiger, Estévez, et al., 2013). In addition, interactive virtual feedback may contribute to more active task-involvement (Kizony, Raz, Katz, Weingarden, \& Weiss, 2005), and to higher levels of enjoyment, motivation, and attention (Villiger, Bohli, et al., 2013; Zimmerli, Jacky, Lünenburger, Riener, \& Bolliger, 2013).

Motor imagery ability as assessed with the KVIQ was found to be variable across participants. This is not surprising, given that we employed a heterogeneous sample (i.e. demographics, SCI characteristics, pharmacological treatment). Still, the mean total 
score and variability observed in our study sample $(30 \pm 12)$ were comparable to KVIQ scores sampled from healthy subjects $(34.8 \pm 7.0, n=47)$ (Malouin et al., 2007). Interestingly, higher general motor imagery ability (i.e. a high KVIQ score) was not associated with better gait imagery performance, and vice versa. For example, one participant (ID1) had a very low KVIQ score (10) but reported high vividness ratings during virtual walking (FWA: 73/100). As such, task-related assessments of motor imagery ability might be preferable to general motor imagery assessment in studies aiming to tailor motor imagery tasks and visual feedback for therapeutic purposes.

\subsection{Interactive virtual feedback did not change neuropathic pain}

The present study did not find any consistent immediate effects of motor imagery with virtual feedback on at-level or below-level neuropathic pain when comparing pre-session to post-session assessments. This contrasts with a previous study in individuals with SCI having at-level neuropathic pain, that reported immediate reductions in pain intensity after only 10 minutes of virtual walking (Moseley, 2007) or with another study in healthy controls where the presence of visual body or movement feedback lead to immediate increases in experimental pain thresholds (Longo, Iannetti, Mancini, Driver, \& Haggard, 2012). Although these contrasting results could be related to the inclusion of individuals with belowlevel pain in the present study, this seems unlikely given the reported long-term beneficial effects of motor imagery on both at-level and below-level pain in multi-session studies (Kumru et al., 2013; Soler et al., 2010; Sumitani et al., 2008; Villiger, Bohli, et al., 2013). Unfortunately these latter multi-session studies did not report on immediate effects within each session. Moreover, in some studies motor imagery was combined with tDCS, and this combination seemed to be associated with additional reductions in pain (Kumru et al., 2013; Soler et al., 2010). The present study also initially aimed at assessing the impact of a combination of virtual walking and tDCS. Unfortunately this was not possible due to difficulties in recruitment (resulting in a limited number of participants). Although we did not observe a difference or trend for a difference between tDCS polarities, we cannot rule out that tDCS had an impact on the present results. Therefore the immediate effect of motor imagery on neuropathic pain intensity, its potential working mechanisms, and its relation to long-term therapeutic efficacy in individuals with SCI remains currently unclear.

\section{Conclusions}

In contrast to previous non - interactive virtual walking protocols, this exploratory study successfully implemented an interactive virtual feedback protocol and showed that interactive control over a full-body avatar was associated with better motor imagery performance as compared to a mere static presentation of the virtual scene. The response to motor imagery interventions after SCI might be improved by using interactive virtual feedback.

\section{Acknowledgments}

The authors are thankful to Dr. Isabelle Côté, France Pelletier, and Martin Gagné for their assistance in outpatient recruitment. MR was supported by a postdoctoral fellowship from the Fonds de recherche Québec - Santé (FRQS) and the SensoriMotor Rehabilitation Research Team as part of the Regenerative Medicine, and the Nanomedicine Strategic Initiative of the Canadian Institute for Health Research (CIHR, RMF-111622). PLJ and CM were supported by salary awards from FRQS and CIHR.

\section{Supplementary material}

Supplementary material can be found here: http:// dx.doi.org/10.3233/RNN-150563

\section{References}

Baumbauer, K.M., Young, E.E., \& Joynes, R.L. (2009). Pain and learning in a spinal system: Contradictory outcomes from common origins. Brain Research Reviews, 61, 124-143.

Beaumont, G., Mercier, C., Michon, P.E., Malouin, F., \& Jackson, P.L. (2011). Decreasing Phantom Limb Pain Through Observation of Action and Imagery: A Case Series. Pain Medicine, 12, 289-299.

Betker, A.L., Desai, A., Nett, C., Kapadia, N., \& Szturm, T. (2007). Game-based exercises for dynamic short-sitting balance rehabilitation of people with chronic spinal cord and traumatic brain injuries. Physical Therapy, 87, 1389-1398.

Boudreau, S., Romaniello, A., Wang, K., Svensson, P., Sessle, B. J., \& Arendt-Nielsen, L. (2007). The effects of intra-oral pain on motor cortex neuroplasticity associated with short-term 
novel tongue-protrusion training in humans. Pain, 132, 169-178.

Bouffard, J., Bouyer, L.J., Roy, J.S., \& Mercier, C. (2014). Tonic Pain Experienced during Locomotor Training Impairs Retention Despite Normal Performance during Acquisition. The Journal of Neuroscience, 34, 9190-9195.

Bowering, K.J., O’Connell, N.E., Tabor, A., Catley, M.J., Leake, H.B., Moseley, G.L., \& Stanton, T.R. (2013). The effects of graded motor imagery and its components on chronic pain: A systematic review and meta-analysis. The Journal of Pain, 14, 3-13.

Decety, J. (1996). The neurophysiological basis of motor imagery. Behavioural Brain Research, 77, 45-52.

Di Rienzo, F., Collet, C., Hoyek, N., \& Guillot, A. (2014). Impact of neurologic deficits on motor imagery: A systematic review of clinical evaluations. Neuropsychology Review, 24, 116-147.

Donnelly, C., \& Eng, J.J. (2005). Pain following spinal cord injury: The impact on community reintegration. Spinal Cord, 43, 278-282.

Gustin, S.M., Wrigley, P.J., Gandevia, S.C., Middleton, J.W., Henderson, L.A., \& Siddall, P.J. (2008). Movement imagery increases pain in people with neuropathic pain following complete thoracic spinal cord injury. Pain, 137, 237-244.

Jackson, P.L., Lafleur, M.F., Malouin, F., Richards, C., \& Doyon, J. (2001). Potential role of mental practice using motor imagery in neurologic rehabilitation. Archives of Physical Medicine and Rehabilitation, 82, 1133-1141.

Jeannerod, M. (2001). Neural simulation of action: A unifying mechanism for motor cognition. NeuroImage, 14, S103-S109.

Kizony, R., Raz, L., Katz, N., Weingarden, H., \& Weiss, P.L. (2005). Video-capture virtual reality system for patients with paraplegic spinal cord injury. Journal of Rehabilitation Research \& Development, 42, 595-608.

Kumru, H., Soler, D., Vidal, J., Navarro, X., Tormos, J.M., PascualLeone, A., \& Valls-Sole, J. (2013). The effects of transcranial direct current stimulation with visual illusion in neuropathic pain due to spinal cord injury: An evoked potentials and quantitative thermal testing study. European Journal of Pain, 17, 55-66.

Lamothe, M., Roy, J.S., Bouffard, J., Gagné, M., Bouyer, L.J., \& Mercier, C. (2014). Effect of Tonic Pain on Motor Acquisition and Retention while Learning to Reach in a Force Field. PLoS ONE, 9, e99159.

Longo, M.R., Iannetti, G.D., Mancini, F., Driver, J., \& Haggard, P. (2012). Linking pain and the body: Neural correlates of visually induced analgesia. The Journal of Neuroscience, 32, 2601-2607.

Malouin, F., \& Richards, C.L. (2010). Mental practice for relearning locomotor skills. Physical Therapy, 90, 240-251.

Malouin, F., Richards, C.L., Jackson, P.L., Lafleur, M.F., Durand, A., \& Doyon, J. (2007). The Kinesthetic and Visual Imagery Questionnaire (KVIQ) for assessing motor imagery in persons with physical disabilities: A reliability and construct validity study. Journal of Neurologic Physical Therapy, 31, 20-29.

Malouin, F., Saimpont, A., Jackson, P.L., \& Richards, C.L. (2013). Promoting locomotor recovery through motor imagery. Movement \& Sport Sciences - Science \& Motricité 129-141.

Mercier, C. (2012). Interactions between motor imagery and pain. Comment on Raffin et al. (2012). Cortex, 48, 778-780.

Mercier, C., \& Sirigu, A. (2009). Training with virtual visual feedback to alleviate phantom limb pain. Neurorehabilitation and Neural Repair, 23, 587-594.

Moseley, G.L. (2006). Graded motor imagery for pathologic pain: A randomized controlled trial. Neurology, 67, 2129-2134.

Moseley, G.L. (2007). Using visual illusion to reduce at-level neuropathic pain in paraplegia. Pain, 130, 294-298.

Moseley, G.L., \& Flor, H. (2012). Targeting cortical representations in the treatment of chronic pain: A review. Neurorehabilitation and Neural Repair, 26, 646-652.

Moseley, G.L., Gallace, A., \& Spence, C. (2012). Bodily illusions in health and disease: Physiological and clinical perspectives and the concept of a cortical 'body matrix'. Neuroscience \& Biobehavioral Reviews, 36, 34-46.

Mulder, T. (2007). Motor imagery and action observation: Cognitive tools for rehabilitation. Journal of Neural Transmission, $114,1265-1278$.

Raffin, E., Giraux, P., \& Reilly, K.T. (2013). The moving phantom: Motor execution or motor imagery? Cortex, 48, 746-757.

Roosink, M., \& Mercier, C. (2014). Virtual feedback for motor and pain rehabilitation after spinal cord injury. Spinal Cord, 52, 860-866.

Sayenko, D.G., Alekhina, M.I., Masani, K., Vette, A.H., Obata, H., Popovic, M.R., \& Nakazawa, K. (2010). Positive effect of balance training with visual feedback on standing balance abilities in people with incomplete spinal cord injury. Spinal Cord, 48, 886-893.

Sharp, K.G., Gramer, R., Butler, L., Cramer, S.C., Hade, E., \& Page, S.J. (2014). Effect of overground training augmented by mental practice on gait velocity in chronic, incomplete spinal cord injury. Archives of Physical Medicine and Rehabilitation, 95, 615-621.

Siddall, P.J., McClelland, J.M., Rutkowski, S.B., \& Cousins, M.J. (2003). A longitudinal study of the prevalence and characteristics of pain in the first 5 years following spinal cord injury. Pain, 103, 249-257.

Soler, M.D., Kumru, H., Pelayo, R., Vidal, J., Tormos, J.M., Fregni, F., et al. (2010). Effectiveness of transcranial direct current stimulation and visual illusion on neuropathic pain in spinal cord injury. Brain, 133, 2565-2577.

Sumitani, M., Miyauchi, S., McCabe, C.S., Shibata, M., Maeda, L., Saitoh, Y., et al. (2008). Mirror visual feedback alleviates deafferentation pain, depending on qualitative aspects of the pain: A preliminary report. Rheumatology, 47, 1038-1043.

Tawashy, A.E., Eng, J.J., Lin, K.H., Tang, P.F., \& Hung, C. (2009). Physical activity is related to lower levels of pain, fatigue and depression in individuals with spinal-cord injury: A correlational study. Spinal Cord, 47, 301-306.

Turner, J.A., Cardenas, D.D., Warms, C.A., \& McClellan, C.B. (2001). Chronic pain associated with spinal cord injuries: A community survey. Archives of Physical Medicine and Rehabilitation, 82, 501-509.

Villiger, M., Bohli, D., Kiper, D., Pyk, P., Spillmann, J., Meilick, B., et al. (2013). Virtual reality-augmented neurorehabilitation 
improves motor function and reduces neuropathic pain in patients with incomplete spinal cord injury. Neurorehabilitation and Neural Repair, 27, 675-683.

Villiger, M., Estévez, N., Hepp-Reymond, M.C., Kiper, D., Kollias, S.S., Eng, K., \& Hotz-Boendermaker, S. (2013). Enhanced Activation of Motor Execution Networks Using Action Observation Combined with Imagination of Lower Limb Movements. PLOS ONE, 8, e72403.

Widerstrom-Noga, E., Biering-Sorensen, F., Bryce, T.N., Cardenas, D.D., Finnerup, N.B., Jensen, M.P., et al. (2014). The International Spinal Cord Injury Pain Basic Data Set (version 2.0). Spinal Cord, 52, 282-286.
Witmer, B.G., \& Singer, M.J. (1998). Measuring presence in virtual environments: A presence questionnaire. Presence: Teleoperators \& Virtual Environments, 7, 225-240.

Zigmond, A.S., \& Snaith, R.P. (1983). The hospital anxiety and depression scale. Acta Psychiatrica Scandinavica, 67, 361370.

Zimmerli, L., Jacky, M., Lünenburger, L., Riener, R., \& Bolliger, M. (2013). Increasing patient engagement during virtual reality-based motor rehabilitation. American Journal of Physical Medicine \& Rehabilitation, 94, 1737-1746. 